\title{
An optimization principle for the computation of MHD equilibria in the solar corona
}

\author{
T. Wiegelmann ${ }^{1}$ and T. Neukirch ${ }^{2}$ \\ 1 Max-Planck-Institut für Sonnensystemforschung, Max-Planck-Strasse 2, 37191 Katlenburg-Lindau, Germany \\ e-mail: wiegelmann@mps.mpg.de \\ 2 School of Mathematics and Statistics, University of St. Andrews, St. Andrews, KY16 9SS, UK
}

Received 27 March 2006 / Accepted 22 June 2006

ABSTRACT

\begin{abstract}
Aims. We develop an optimization principle for computing stationary MHD equilibria.
Methods. Our code for the self-consistent computation of the coronal magnetic fields and the coronal plasma uses non-force-free MHD equilibria. Previous versions of the code have been used to compute non-linear force-free coronal magnetic fields from photospheric measurements. The program uses photospheric vector magnetograms and coronal EUV images as input. We tested our reconstruction code with the help of a semi-analytic MHD-equilibrium. The quality of the reconstruction was judged by comparing the exact and reconstructed solution qualitatively by magnetic field-line plots and EUV-images and quantitatively by several different numerical criteria.

Results. Our code is able to reconstruct the semi-analytic test equilibrium with high accuracy. The stationary MHD optimization code developed here has about the same accuracy as its predecessor, a non-linear force-free optimization code. The computing time for MHD-equilibria is, however, longer than for force-free magnetic fields. We also extended a well-known class of nonlinear force-free equilibria to the non-force-free regime for purposes of testing the code.

Conclusions. We demonstrate that the code works in principle using tests with analytical equilibria, but it still needs to be applied to real data.
\end{abstract}

Key words. Sun: magnetic fields - Sun: corona - Sun: photosphere - magnetohydrodynamics (MHD)

\section{Introduction}

Understanding many physical phenomena in the solar corona requires detailed knowledge of the properties of the coronal magnetic field and plasma. Usually neither the coronal magnetic field nor the plasma density, pressure, temperature, and flow speed are directly observable. Direct observations of the coronal magnetic field are difficult because the high temperature broadens the line-profile orders of magnitude above the Zeeman splitting. The optically-thin coronal line emission has a line-of-sight integrated character, which complicates the computation of any plasma quantities. A way out of this dilemma is the use of coronal models, which are fed with as much observations as possible. The model assumptions, e.g. an MHD model, constrains the nonobserved quantities. Often it is not the full set of MHD equations used, but a subset.

The simplest approach for coronal magnetic field calculations is to assume a potential magnetic field (e.g. Schmidt 1964; Semel 1967). The only observational input required is the line-of-sight photospheric magnetic field, such as observed by SOHO/MDI. These source surface potential field models provide a first impression regarding the global coronal magnetic field, e.g. regarding the location of coronal holes and active regions. Details of the magnetic field structure are often not well-approximated by potential field models, particularly in active regions. Approaches using force-free magnetic fields (with electric currents parallel to the magnetic field) show results which are significantly better. The low plasma beta in the corona $\left(\beta \approx 10^{-4}\right)$ justifies that approach in the low and middle corona, but not in the photosphere $(\beta \approx 1)$ where non magnetic forces like pressure gradients become important. A popular simplification of force-free fields are linear force-free fields (e.g. Chiu \& Hilton 1977; Seehafer 1978)) where the electric current flow is parallel to the magnetic field with a global constant of proportionality $\alpha$.

A more sophisticated approach is to allow $\alpha$ to change in space, the so-called nonlinear force-free approach. The calculation of non-linear force-free fields (e.g. Sakurai 1981; Wu et al. 1990; Wheatland et al. 2000; Yan \& Sakurai 2000; Régnier et al. 2002; Wiegelmann \& Neukirch 2003; Wiegelmann 2004; Wheatland 2004; Valori et al. 2005; Amari et al. 2006; Wiegelmann et al. 2006; Inhester \& Wiegelmann 2006; Schrijver et al. 2006) is particularly challenging due to the intrinsic nonlinearity of the underlying mathematical problem. From an observational point of view the nonlinear reconstruction is also more demanding because photospheric vector magnetograph data are required to determine the boundary conditions.

A comparison of the magnetic loops measured by Solanki et al. (2003) and Lagg et al. (2004) with different extrapolated field models by Wiegelmann et al. (2005b) revealed that linear force-free fields are better than potential fields, but non-linear force-free models are even more accurate. Usually the coronal magnetic field cannot be measured directly, also some progress has been made recently by using magnetically sensitive coronal line emission, e.g., by Lin et al. (2004) who did spectropolarimetric measurements of the forbidden Fe XIII $1075 \mathrm{~nm}$ infrared coronal line. The influence of the coronal magnetic field onto the emitting plasma is however observed routinely. 
Images of the coronal plasma emission are obtained by the radiation in different wave lengths, e.g. in EUV for SOHO/EIT and TRACE. The emission is obtained from various elements, e.g. Fe XII or Fe IX, with different instrument channels sensitive to emission generated at different plasma temperatures. The corresponding images have a line-of-sight character because of the corona is optically thin. A rather good approximation of the coronal temperature is sometimes possible, because the radiation only occurs in a specific temperature range. Doppler shifts in the line profile observed with e.g. SOHO/SUMER provide insight into the plasma flow speed in the line-of-sight direction. The high electrical conductivity of the coronal plasma ensures that the plasma is frozen into the magnetic field. This basically allows us to see (the effects of) magnetic field lines in EUV-images and even use the visible plasma radiation to improve coronal magnetic field models, e.g. to specify the optimal value $\alpha$ for linear force-free models (see e.g. Wiegelmann \& Neukirch 2002; Carcedo et al. 2003; Marsch et al. 2004; Wiegelmann et al. 2005a).

For the low $\beta$ corona, it is also helpful that the back reaction of the plasma onto the magnetic field can be neglected. Marsch et al. (2004) used a linear force-free coronal magnetic field model and Doppler maps from SOHO/SUMER to investigate the plasma flow in active regions. Wiegelmann et al. (2005c) and Tu et al. $(2005 \mathrm{a}, \mathrm{b})$ used SUMER Doppler maps with potential and linear force-free models to study the outflow of the solar wind in coronal holes. The next natural step is to use more sophisticated magnetic field models, where the magnetic field and plasma to compute self-consistently in one model, as proposed here. In this paper we first present the basic equations used in Sect. 2 (supplemented by a couple of appendices). We then present the algorithm based on the equations (Sect. 3) and the derivation of the non-force-free MHD equilibria we used for testing the code (Sect. 4). The results are presented in Sect. 5, followed by a discussion and conclusions in Sect. 6 .

\section{Basic equations}

The MHD equilibrium equations (here without gravity for simplicity $^{1}$ ) are

$$
\begin{aligned}
(\nabla \times \boldsymbol{B}) \times \boldsymbol{B}-\mu_{0} \nabla p & =\mathbf{0} \\
\nabla \cdot \boldsymbol{B} & =0 .
\end{aligned}
$$

We have

$\boldsymbol{B} \cdot \nabla p=0$,

i.e. the pressure is constant along magnetic field lines. We show in Appendix A that it is possible in principle to include fieldaligned incompressible flows into the method. We take this possibility into account by replacing the plasma pressure $p$ by a generalized pressure $\Pi=p+\rho v^{2} / 2$ from now on. We did not, however, calculate any example cases with field-aligned flow, because one would need more information to disentangle the

\footnotetext{
${ }^{1}$ While it is, in principle, possible to include gravity in the optimization principle, it is hard to find (semi)-analytic equilibria to test the code. For configurations that are small compared to the gravity scale height of some 0.1 solar radii, gravity might be neglected in first order, however. A consistent treatment of large-scale (some solar radii) structures, like helmet streamers, require not only the inclusion of plasma pressure and gravity, but also the use of spherical geometry, see e.g., Wiegelmann et al. (1998), but such computations are well beyond the scope of this paper.
}

contributions of the plasma pressure and the energy density of the flow.

The general form of the MHD equilibrium equations is given by

$(\nabla \times \boldsymbol{B}) \times \boldsymbol{B}=\nabla \Lambda$

$\nabla \cdot \boldsymbol{B}=0$

with the special cases

$\Lambda= \begin{cases}p_{0}=\text { const. } & \text { (force }- \text { free equilibria) } \\ \nabla\left(\mu_{0} p\right) & (\text { MHS equilibria) } \\ \nabla\left(\frac{\mu_{0} \Pi}{1-M_{\mathrm{A}}^{2}}\right) & \left(\text { field }- \text { aligned flow, constant } M_{\mathrm{A}}\right)\end{cases}$

To solve Eqs. (4) and (5), we define the functional

$L(\boldsymbol{B}, \Lambda)=\int \frac{w_{a}}{B^{2}}|(\nabla \times \boldsymbol{B}) \times \boldsymbol{B}-\nabla \Lambda|^{2}+w_{b}|\nabla \cdot \boldsymbol{B}|^{2} \mathrm{~d}^{3} x$

where $w_{a}$ and $w_{b}$ are positive definite weighting functions ${ }^{2}$.

It is obvious that Eqs. (4) and (5) are satisfied if the functional (7) reaches its minimum at $L=0$. The functional (7) generalizes the force-free approach of Wheatland et al. (2000) and the magnetohydrostatic model of Wiegelmann \& Inhester (2003).

To obtain evolution equations for the magnetic field and the generalized plasma pressure, we take the derivative of Eq. (7) with respect to an artificial parameter $t$, assuming that both $\boldsymbol{B}$ and $\Lambda$ depend on $t$ :

$$
\begin{aligned}
\frac{1}{2} \frac{\mathrm{d} L}{\mathrm{~d} t}= & -\int_{V} \frac{\partial \boldsymbol{B}}{\partial t} \cdot \tilde{\boldsymbol{F}}-\nabla \cdot \boldsymbol{\Omega}_{a} \frac{\partial \Lambda}{\partial t} \mathrm{~d}^{3} x \\
& -\int_{S} \frac{\partial \boldsymbol{B}}{\partial t} \cdot \tilde{\boldsymbol{G}}+\boldsymbol{\Omega}_{a} \cdot \hat{\boldsymbol{n}} \frac{\partial \Lambda}{\partial t} \mathrm{~d}^{2} x
\end{aligned}
$$

where $\tilde{\boldsymbol{F}}$ and $\tilde{\boldsymbol{G}}$ are defined in Appendix B.

If $\boldsymbol{B}$ and $\Lambda$ are kept fixed on the boundary of the computational box, the surface integral vanishes and we can minimize $L$ by the solving the equations

$$
\begin{aligned}
& \frac{\partial \boldsymbol{B}}{\partial t}=\mu \tilde{\boldsymbol{F}} \\
& \frac{\partial \Lambda}{\partial t}=-v \nabla \cdot \boldsymbol{\Omega}_{a}
\end{aligned}
$$

iteratively, with positive constants $\mu$ and $v$ (see Appendix B for the mathematical derivation). The form of Eqs. (9) and (10) ensures that $L$ decreases monotonically during the computation of $\boldsymbol{B}$ and $\Lambda$.

\section{Algorithm}

To compute nonlinear, selfconsistent 3D-MHD equilibria, we use the following steps:

1. Compute a potential field. This can be done from the $B_{z}$ component of the vector magnetogram alone.

2. Distribute the plasma (or, say, the generalized plasma pressure $\Lambda$ ) along the potential magnetic field by solving

$$
\boldsymbol{B} \cdot \nabla \Lambda=0
$$

2 The functions $w_{a}$ and $w_{b}$ can e.g. be used to deal with unknown boundary conditions (see Wiegelmann 2004). To test the method, it is sufficient to use $w_{a}=w_{b}=1$. 
with an upwind method (used here) or a magnetic field line tracer $^{3}$.

3. Substitute the boundary values of the computational box from the observed vector magnetogram. The interior of the computational box remains filled with a potential field and a corresponding plasma distribution.

4. Iterate for $\boldsymbol{B}$ and $\Lambda$ by Eqs. (9) and (10). The continuous form of these equations ensures that $L$ is monotonically decreasing. This is also ensured in the discretized form if the iteration step $\mathrm{d} t$ is small enough. The code automatically controls the optimal iteration step. If $L(t+\mathrm{d} t) \geq L(t)$, the step is refused and repeated with $\mathrm{d} t$ reduced by a factor of two. We increase $\mathrm{d} t$ by a factor 1.01 after each successful iteration step to allow $\mathrm{d} t$ to be as large as possible with respect to the stability condition.

5. The iteration stops when $L$ reaches its minimum. In practise we stop the iteration when $\frac{\partial L}{\partial t} / L<10^{-6}$ for 100 consecutive steps.

6. As result we get the magnetic field $\boldsymbol{B}$ and generalized plasma pressure $\Lambda$, which fulfill the MHD equations and is consistent with the observed boundary conditions. In the final step one has to disentangle the generalized plasma pressure $\Lambda$ with respect to the plasma pressure $p$ and the flow velocity $v$. For MHS equilibria, this is trivial: $p=\frac{\Lambda}{\mu_{0}}$; for equilibria with flow one needs further observations/assumptions regarding the plasma flow, e.g. from SOHO/SUMER.

\section{Semi-analytic test equilibrium}

We test our method with the help of a semi analytic equilibrium similar to the force-free Low \& Lou (1990) solution (henceforth LL). We solve the Grad-Shafranov equation for axissymmetric force-free fields in spherical coordinates $r, \theta, \phi$ :

$-\frac{\partial^{2} A}{\partial r^{2}}-\frac{1-\mu^{2}}{r^{n}} \frac{\partial^{2} A}{\partial \mu^{2}}=\mu_{0} r^{2}\left(1-\mu^{2}\right) \frac{\mathrm{d} \Lambda}{\mathrm{d} A}+b_{\phi} \frac{\mathrm{d} b_{\phi}}{\mathrm{d} A}$.

LL looked for self-similar (in $r$ ) solutions by choosing $b_{\phi}(a)=$ $c A^{q}$. We generalize this approach by also choosing $\Lambda(A)=k A^{s}$, where the power $s$ has to be chosen such that self-similar solutions are possible.

Following LL we assume

$A(r, \mu)=\frac{P(\mu)}{r^{n}}$.

Substituting this into Eq. (12) and using the above expressions for $\Lambda$ and $b_{\phi}$, we get

$$
\begin{aligned}
-n(n+1) & \frac{P}{r^{n+2}}-\frac{1-\mu^{2}}{r^{n+2}} \frac{d^{2} P}{d \mu^{2}}= \\
& \mu_{0} s k\left(1-\mu^{2}\right) \frac{P^{s-1}}{r^{n(s-1)-2}}+q c \frac{P^{2 q-1}}{r^{n(2 q-1)}} .
\end{aligned}
$$

We now determine $q$ and $s$ such that all powers of $r$ are equal, obtaining the equations

$n+2=n(s-1)-2$

$n+2=n(2 q-1)$.

${ }^{3}$ By multiplying Eq. (6) with $\boldsymbol{B}$, we get $\boldsymbol{B} \cdot \nabla \Lambda=0$, which implies that the generalized pressure $\Lambda$ is constant on magnetic field lines. This kind of equation $\boldsymbol{B} \cdot \nabla \alpha=0$ is also used in Grad-Rubin like extrapolation codes to distribute the force-free parameter $\alpha$ along the field lines in space.
Solving Eq. (15) gives

$s=2+\frac{4}{n}$,

whereas Eq. (16) gives the same result as LL

$q=1+\frac{1}{n}$

The equation for $P(\mu)$ is then given by

$$
\begin{aligned}
\left(1-\mu^{2}\right) \frac{d^{2} P}{d \mu^{2}}+n(n+1) P & +2 k\left(1+\frac{2}{n}\right)\left(1-\mu^{2}\right) P^{1+4 / n} \\
& +a^{2}\left(1+\frac{1}{n}\right) P^{1+2 / n}=0 .
\end{aligned}
$$

This equation is nonlinear and has to be solved numerically.

For our test equilibrium, we use a similar parameter set to the one in LL for the force-free case ( $\left.\Phi=\frac{\pi}{4}, l=0.3\right)$. In Eq. (19) we choose (as LL) $a^{2}=0.425$. Both $\Phi$ and $l$ have the same meaning as in LL here.

The difference of our solution to LL is, that we have the additional term $2 k\left(1+\frac{2}{n}\right)\left(1-\mu^{2}\right) P^{1+4 / n}$ which corresponds to the (generalised) plasma pressure. ( $k=0$ corresponds to LL.) For the present paper we choose $k=10$ and $\frac{\partial p}{\partial \mu}=2.097$ for $\mu=-1$.

\section{Results}

In Fig. 1 we compare some magnetic field lines of the original solution (left panel) with the result of our reconstruction (right panel). The reconstructed solution obviously agrees well with the original, and an inspection by eye only shows hardly visible differences for some loops. Figure 2 contains images produced from the original (left panels) and reconstructed (right panels) plasma pressure. The images have been produced by a line-of-sight integration along the $z$-axis to mimic the optically thin coronal plasma. The top panels use a linear scaling and the bottom panels a logarithmic one. While the overall structure of the plasma emission agrees in the original and the reconstruction, there are small deviations (visible in the logarithmic images) in darker (weak magnetic field) regions, in particular at the right side of the image. To evaluate the quality of reconstruction we used several figures of merit. The functional $L$ as defined in Eq. (7) provides automatically a measure of how well the MHD equilibria and the solenoidal condition are satisfied.

The evolution of $L$ during the optimization process is shown in Fig. 3 as a function of the iteration steps. For the $64^{3}$ box, $L$ has decreased below the discretization error of the analytic solution during the iteration. The number of iteration steps (and thus the computing time) for convergence is, however, about 1000 times greater than for a corresponding force-free optimization. The reason for this behavior is because the semi-analytic solution has a huge difference in magnitude of the pressure throughout the computational box with $p_{\max }=1.9133 \times 10^{4}$ and $p_{\text {min }}=1.0239 \times 10^{-21}$. The code obviously has problems dealing with the pressure differences during the iteration. Test runs with prescribed pressure profile (only iterating for $\boldsymbol{B}$ ) converge at the same rate as a corresponding force-free calculation (see e.g., Schrijver et al. 2006, where the magnetic pressure $\frac{B^{2}}{2}$ varies between $4.616 \times 10^{4}$ and $2.87 \times 10^{-2}$ ).

Schrijver et al. (2006) developed several figures of merit to quantify the difference between two vector fields $\boldsymbol{B}$ (semianalytic test field) and $\boldsymbol{b}$ (reconstructed fields). The figures have 

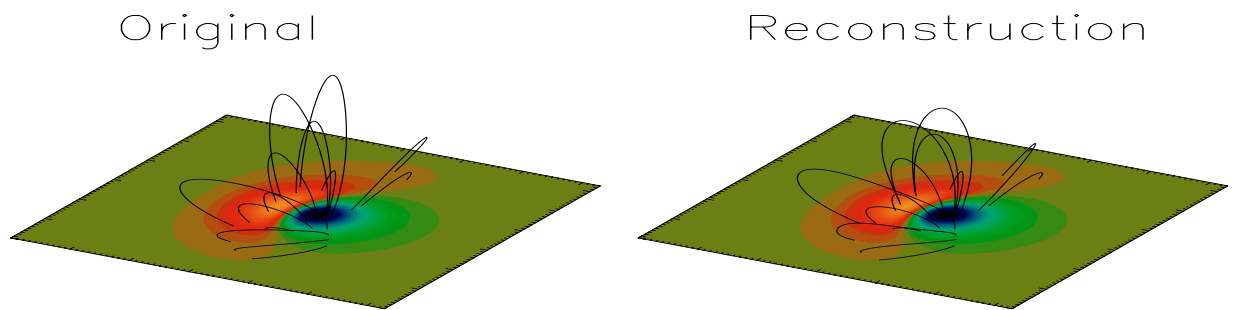

Fig. 1. Left panel: original MHD-equilibrium; right panel: reconstruction. The colour coding shows the line-of sight magnetic field on the photosphere.
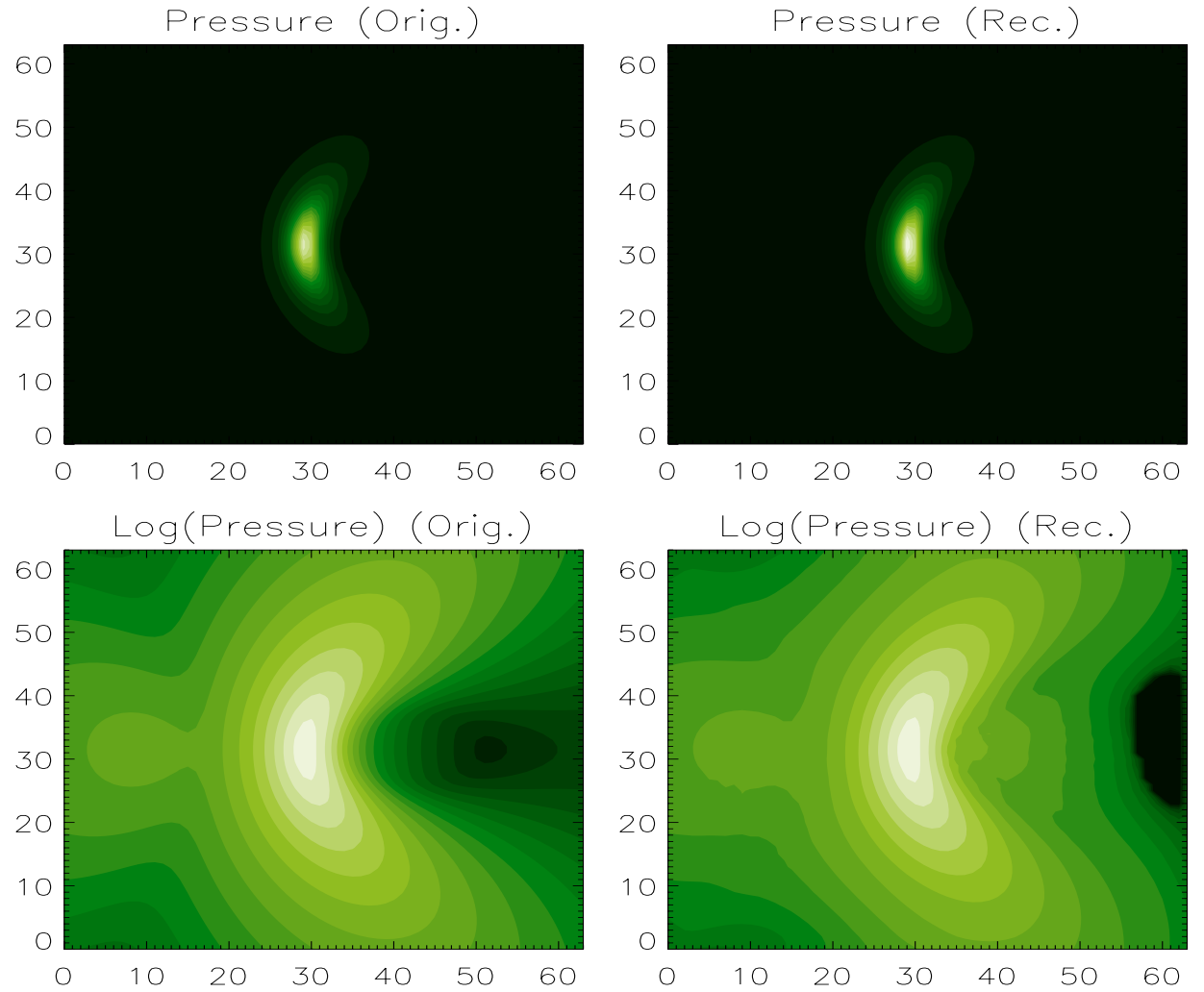

Fig. 2. We show the line-of-sight integration of $\Lambda$ along the $z$-axis (top: linear scaling, bottom logarithmic scaling). This quantity seems to be important because coronal images have a line-of-sight integrated character as a consequence of the optical thin coronal line emission. The left hand panels correspond to the original solution and the right hand panels to our reconstruction.

been used to evaluate the quality of six different non-linear forcefree extrapolation codes, by comparing the result with the LL solution. We use the same definitions as given in Sect. 4 of Schrijver et al. (2006) for evaluating the quality of the non forcefree magnetic fields here:

- vector correlation

$$
C_{\mathrm{vec}}=\sum_{i} \boldsymbol{B}_{i} \cdot \boldsymbol{b}_{i} /\left(\sum_{i}\left|\boldsymbol{B}_{i}\right|^{2} \sum_{i}\left|\boldsymbol{b}_{i}\right|^{2}\right)^{1 / 2} ;
$$

- Cauchy-Schwarz inequality

$$
C_{\mathrm{CS}}=\frac{1}{N} \sum_{i} \frac{\boldsymbol{B}_{i} \cdot \boldsymbol{b}_{i}}{\left|\boldsymbol{B}_{i} \| \boldsymbol{b}_{i}\right|}
$$

where $N$ is the number of vectors in the field;

- normalized vector error

$$
E_{\mathrm{N}}=\sum_{i}\left|\boldsymbol{b}_{i}-\boldsymbol{B}_{i}\right| / \sum_{i}\left|\boldsymbol{B}_{i}\right|
$$

- mean vector error

$$
E_{\mathrm{M}}=\frac{1}{N} \sum_{i} \frac{\left|\boldsymbol{b}_{i}-\boldsymbol{B}_{i}\right|}{\left|\boldsymbol{B}_{i}\right|}
$$

- magnetic energy of the reconstructed field normalized with the energy of the input field

$$
\epsilon=\frac{\sum_{i}\left|\boldsymbol{b}_{i}\right|^{2}}{\sum_{i}\left|\boldsymbol{B}_{i}\right|^{2}}
$$

The two vector fields agree perfectly if the figures of merit $\left(C_{\mathrm{vec}}, C_{\mathrm{CS}}, \epsilon\right)$ are unity and if $\left(E_{N}, E_{M}\right)$ are zero.

We also compare how closely the generalized plasma pressure $\Lambda$ of our reconstruction agrees with the original. To do so we compute the linear Pearson correlation coefficient, both for the 3D plasma pressure $\operatorname{Corr} \Lambda_{3 D}$ and the line-of-sight integration (with respect to the $z$ axis) $\operatorname{Corr} \Lambda_{2 D}$. The latter value is a useful quantity because observed EUV-images (e.g. SOHO/EIT, TRACE) have a line-of sight integrated character as a consequence of the optically thin coronal plasma. 


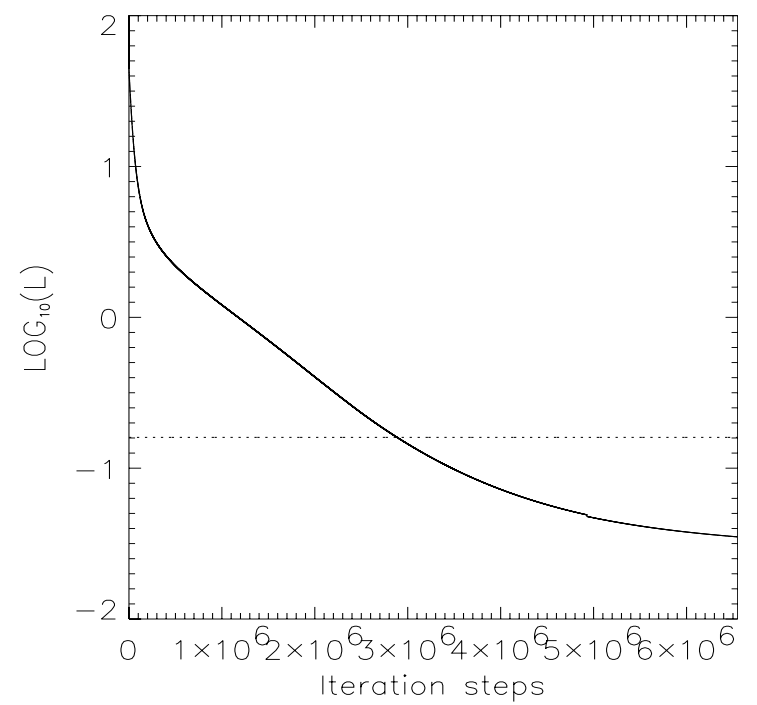

Fig. 3. Evolution of the functional $L(\boldsymbol{B}, N)$ during the iteration for a box of $64^{3}$. The dotted line corresponds to the discretisation error of the analytic solution $\left[L=0.16\right.$ or $\left.\log _{10}(L)=-0.80\right]$.

\section{Discussion and conclusions}

We have generalized the optimization method for nonlinear force-free fields (Wheatland et al. 2000) to magnetohydrostatic equilibria by including a pressure gradient. Using a semianalytical magnetohydrostatic equilibrium similar to the nonlinear force-free equilibria by Low \& Lou (1990) for testing the code, we showed that the optimization method also works in principle if plasma pressure is included. The reconstructed solution agrees well with the exact solution. For application to real data, the method will have to be developed further to include measured information about the plasma properties, which in the present paper we have taken from the exact solution. We have also shown that it is possible to include an incompressible fieldaligned plasma flow.

Compared to the corresponding nonlinear force-free code, however, the MHD-optimization method presented here (for $\boldsymbol{B}$ and $\Lambda$ simultaneously) is about a factor of $10^{3}$ slower than forcefree computation. The reason for this seems to be the additional equation for updating the plasma pressure. If we prescribe the correct exact pressure and solve only for the magnetic field, the convergence speed of the method is similar to that of the corresponding force-free case. If, on the other hand, we fix the magnetic field using the exact solution and iterate only the pressure, the convergence speed is much less and comparable to the convergence speed of the combined magnetic field/plasma pressure iteration. The reason for this behavior by the method is the huge difference in the values of the plasma pressure throughout the computational box, combined with the relatively low values of the plasma $\beta$. This means that even small changes in the magnetic field have to compensate for by much larger changes in the plasma pressure.

Despite these practical difficulties, we believe that the method has a lot of potential to improve magnetic-field reconstruction by including more information from observations.

\section{Appendix A: Including field-aligned flow}

For completeness, we give some details here of how fieldaligned incompressible flows could in principle be included in the optimization method. Although flows seem to be common in
Table 1. Quality of the reconstruction.

\begin{tabular}{lcc}
\hline \hline & Exact & Rec. \\
\hline$L$ & 0.16 & 0.035 \\
Vector correlation & 1 & 0.999 \\
Cauchy Schwarz & 1 & 0.992 \\
Normalized vector error & 0 & 0.130 \\
Mean vector error & 0 & 0.073 \\
Relative magnetic energy $\epsilon$ & 1 & 0.996 \\
Corr $\Lambda_{3 \mathrm{D}}$ & 1 & 0.998 \\
Corr $\Lambda_{2 \mathrm{D}}$ & 1 & 0.999 \\
\hline
\end{tabular}

the corona, the inertial force scales with the square of the Alfvén Mach number, which usually means that only flows with Alfvén Mach numbers close to 1 have a noticeable effect upon the equilibrium structure (e.g. Petrie \& Neukirch 1999). We also notice that to use the method including flow one would need additional information, to be able to disentangle the effects of the plasma pressure and plasma flow. The assumption of an incompressible field-aligned flow is an idealization, because any real flow that approaches the base of the coronal loops, which has small pressure scale heights, will be very compressible, even when the flow is slow.

We restrict our treatment here to incompressible fieldaligned flows with constant Alfvén Mach number $M_{\mathrm{A}}$. For incompressible field-aligned flows, a general transformation theory exists (e.g. Gebhardt \& Kiessling 1992) that allows the mapping of equilibria with field-aligned incompressible flow (with $M_{\mathrm{A}}<1$ ) onto static MHD equilibria. For further details we refer to Gebhardt \& Kiessling (1992).

Neglecting gravity for simplicity, the stationary incompressible MHD equations are

$$
\begin{aligned}
\rho(\boldsymbol{v} \cdot \nabla) \boldsymbol{v} & =\frac{1}{\mu_{0}}(\nabla \times \boldsymbol{B}) \times \boldsymbol{B}-\nabla p \\
\nabla \cdot \boldsymbol{B} & =0 \\
\nabla \cdot(\rho \boldsymbol{v}) & =0 \\
\nabla \cdot \boldsymbol{v} & =0 \\
\nabla \times(\boldsymbol{v} \times \boldsymbol{B}) & =0 .
\end{aligned}
$$

Equations (A.3) and (A.4) imply that the plasma density is constant along magnetic field lines:

$\boldsymbol{B} \cdot \nabla \rho=0$.

Equation (A.5) is identically satisfied for field-aligned flow (v\|B, implying a vanishing electric field). The plasma velocity can then be written as

$\boldsymbol{v}=M_{\mathrm{A}} \boldsymbol{v}_{\mathrm{A}}$

where $M_{\mathrm{A}}$ is the Alfvén Mach number and $\boldsymbol{v}_{\mathrm{A}}$ the Alfvén velocity, defined by

$\boldsymbol{v}_{\mathrm{A}}=\frac{\boldsymbol{B}}{\sqrt{\mu_{0} \rho}}$.

Rewriting the force balance equation with the vector identity

$(\boldsymbol{v} \cdot \nabla) \boldsymbol{v}=\frac{1}{2} \nabla v^{2}+(\nabla \times \boldsymbol{v}) \times \boldsymbol{v}$,

it takes the form

$\rho(\nabla \times \boldsymbol{v}) \times \boldsymbol{v}=\frac{1}{\mu_{0}}(\nabla \times \boldsymbol{B}) \times \boldsymbol{B}-\nabla\left(p+\frac{\rho v^{2}}{2}\right)$, 
and for a constant Alfvén Mach number $M_{\mathrm{A}}$ we immediately get

$$
\begin{aligned}
(\nabla \times \boldsymbol{B}) \times \boldsymbol{B} & =\nabla\left(\frac{\mu_{0} \Pi}{1-M_{\mathrm{A}}^{2}}\right) \\
\nabla \cdot \boldsymbol{B} & =0
\end{aligned}
$$

where $\Pi=p+\frac{\rho v^{2}}{2}$ is the generalized pressure (plasma pressure and dynamic pressure). Equation (A.7) has (at least for $M_{\mathrm{A}}<1$ ) a structure that is similar to the magnetohydrostatic equilibrium equation. This is a natural property of MHD equilibria with an incompressible stationary plasma flow, which can be derived from MHS-equilibria by a suitable transformation as shown, for example, in Gebhardt \& Kiessling (1992). Equations (A.7) and (A.8) can then, in principle, be solved using the method presented in Sect. 2, but more information would be needed to obtain the plasma pressure $p$ and the density $\rho$ and velocity $v$ separately.

\section{Appendix B: Mathematical details}

With

$\boldsymbol{\Omega}_{a}=B^{-2}\left[(\nabla \times \boldsymbol{B}) \times \boldsymbol{B}-\nabla\left(\frac{\mu_{0} \Pi}{1-M_{\mathrm{A}}^{2}}\right)\right]$

$\boldsymbol{\Omega}_{b}=B^{-2}[(\nabla \cdot \boldsymbol{B}) \boldsymbol{B}]$,

the functional (7) reads:

$L=\int_{V} w_{a} B^{2} \Omega_{a}^{2}+w_{b} B^{2} \Omega_{b}^{2} \mathrm{~d}^{3} x$.

We minimize Eq. (B.3) with respect to an iteration parameter $t$ and obtain an iteration equation for the magnetic field

$\Rightarrow \frac{1}{2} \frac{\mathrm{d} L}{\mathrm{~d} t}=-\int_{V} \frac{\partial \boldsymbol{B}}{\partial t} \cdot \tilde{\boldsymbol{F}} \mathrm{d}^{3} x-\int_{S} \frac{\partial \boldsymbol{B}}{\partial t} \cdot \tilde{\boldsymbol{G}} \mathrm{d}^{2} x$

$\tilde{\boldsymbol{F}}=\tilde{\boldsymbol{F}}_{a}+\tilde{\boldsymbol{F}}_{b}$

$\tilde{\boldsymbol{G}}=\tilde{\boldsymbol{G}}_{a}+\tilde{\boldsymbol{G}}_{b}$

$\tilde{\boldsymbol{F}}_{a}=w_{a} \boldsymbol{F}_{a}+\left(\boldsymbol{\Omega}_{a} \times \boldsymbol{B}\right) \times \nabla w_{a}$

$\tilde{\boldsymbol{F}}_{b}=w_{b} \boldsymbol{F}_{b}+\left(\boldsymbol{\Omega}_{b} \cdot \boldsymbol{B}\right) \nabla w_{b}$

$\tilde{\boldsymbol{G}}_{a}=w_{a} \boldsymbol{G}_{a}$

$\tilde{\boldsymbol{G}}_{b}=w_{b} \boldsymbol{G}_{b}$

$$
\boldsymbol{F}_{a}=\nabla \times\left(\boldsymbol{\Omega}_{a} \times \boldsymbol{B}\right)-\boldsymbol{\Omega}_{a} \times(\nabla \times \boldsymbol{B})+\Omega_{a}^{2} \boldsymbol{B}
$$

$$
\boldsymbol{F}_{b}=\nabla\left(\boldsymbol{\Omega}_{b} \cdot \boldsymbol{B}\right)-\boldsymbol{\Omega}_{b}(\nabla \cdot \boldsymbol{B})+\Omega_{b}^{2} \boldsymbol{B}
$$

$\boldsymbol{G}_{a}=\hat{\boldsymbol{n}} \times\left(\boldsymbol{\Omega}_{a} \times \boldsymbol{B}\right)$

$\boldsymbol{G}_{b}=-\hat{\boldsymbol{n}}\left(\boldsymbol{\Omega}_{b} \cdot \boldsymbol{B}\right)$

and $\hat{n}$ is the inward unit vector on the surface $S$. The surface integral in (B.4) vanishes if the magnetic field is described on the boundaries of a computational box.

Acknowledgements. The work of Wiegelmann was supported by DLR-grant 50 OC 0501. T.W. acknowledges the warm hospitality during two research visits in the Solar group, university St. Andrews, UK, and financial support by a British Council-DAAD and a PPARC grant.

\section{References}

Amari, T., Boulmezaoud, T. Z., \& Aly, J. J. 2006, A\&A, 446, 691 Carcedo, L., Brown, D. S., Hood, A. W., Neukirch, T., \& Wiegelmann, T. 2003, Sol. Phys., 218, 29

Chiu, Y. T., \& Hilton, H. H. 1977, ApJ, 212, 873

Gebhardt, U., \& Kiessling, M. 1992, Physics of Fluids B, 4, 1689

Inhester, B., \& Wiegelmann, T. 2006, Sol. Phys., 235, 201

Lagg, A., Woch, J., Krupp, N., \& Solanki, S. K. 2004, A\&A, 414, 1109

Lin, H., Kuhn, J. R., \& Coulter, R. 2004, ApJ, 613, L177

Low, B. C., \& Lou, Y. Q. 1990, ApJ, 352, 343

Marsch, E., Wiegelmann, T., \& Xia, L. D. 2004, A\&A, 428, 629

Petrie, G. J. D., \& Neukirch, T. 1999, Geophysical and Astrophysical Fluid Dynamics, 91, 269

Régnier, S., Amari, T., \& Kersalé, E. 2002, A\&A, 392, 1119

Sakurai, T. 1981, Sol. Phys., 69, 343

Schmidt, H. U. 1964, in The Physics of Solar Flares, 107

Schrijver, C. J., Derosa, M. L., Metcalf, T. R., et al. 2006, Sol. Phys., 235, 161

Seehafer, N. 1978, Sol. Phys., 58, 215

Semel, M. 1967, Annales d'Astrophysique, 30, 513

Solanki, S. K., Lagg, A., Woch, J., Krupp, N., \& Collados, M. 2003, Nature, 425, 692

Tu, C.-Y., Zhou, C., Marsch, E., et al. 2005a, ApJ, 624, L133

Tu, C.-Y., Zhou, C., Marsch, E., et al. 2005b, Science, 308, 519

Valori, G., Kliem, B., \& Keppens, R. 2005, A\&A, 433, 335

Wheatland, M. S. 2004, Sol. Phys., 222, 247

Wheatland, M. S., Sturrock, P. A., \& Roumeliotis, G. 2000, ApJ, 540, 1150

Wiegelmann, T. 2004, Sol. Phys., 219, 87

Wiegelmann, T., \& Inhester, B. 2003, Sol. Phys., 214, 287

Wiegelmann, T., \& Neukirch, T. 2002, Sol. Phys., 208, 233

Wiegelmann, T., \& Neukirch, T. 2003, Nonlinear Processes in Geophysics, 10, 313

Wiegelmann, T., Schindler, K., \& Neukirch, T. 1998, Sol. Phys., 180, 439

Wiegelmann, T., Inhester, B., Lagg, A., \& Solanki, S. K. 2005a, Sol. Phys., 228, 67

Wiegelmann, T., Lagg, A., Solanki, S. K., Inhester, B., \& Woch, J. 2005b, A\&A, 433, 701

Wiegelmann, T., Xia, L. D., \& Marsch, E. 2005c, A\&A, 432, L1

Wiegelmann, T., Inhester, B., \& Sakurai, T. 2006, Sol. Phys., 233, 215

Wu, S. T., Sun, M. T., Chang, H. M., Hagyard, M. J., \& Gary, G. A. 1990, ApJ, 362, 698

Yan, Y., \& Sakurai, T. 2000, Sol. Phys., 195, 89 